\title{
DENSIDADE, TAMANHO POPULACIONAL E ABUNDÂNCIA DOS PRIMATAS EM UM FRAGMENTO DE FLORESTA ATLÂNTICA EM MINAS GERAIS, BRASIL ${ }^{1}$
}

\author{
Belmira Evânia Mendes Marques Santana², Maressa Rocha do Prado ${ }^{3}$, Gisele Lessa ${ }^{4}$, Ednaldo Cândido
} Rocha $^{3}$ e Fabiano Rodrigues de Melo ${ }^{5}$

\begin{abstract}
RESUMO - A diversidade de primatas neotropicais está representada por 128 espécies, e no Brasil 26 estão em categorias de ameaça de extinção e 24 são endêmicas. Desses primatas ameaçados, 15 espécies ocorrem na Floresta Atlântica. A Mata do Paraíso, maior fragmento desse bioma em Viçosa, MG, possui área de 384,5 ha, cuja fauna de primatas está representada por Callicebus nigrifrons (Spix, 1823) e Callithrix sp. Este estudo objetivou estimar a densidade e tamanho populacional, bem como determinar a abundância dos primatas na Mata do Paraíso. Para estimar a densidade e tamanho populacional, foram percorridos cinco transectos lineares de $1 \mathrm{~km}$ cada, dispostos paralelamente no interior da mata, seguindo-se as premissas da metodologia Distance para transectos lineares. Os dados foram coletados de agosto de 2004 a fevereiro de 2006, durante 70 levantamentos, totalizando $82,7 \mathrm{~km}$ percorridos e 12 avistamentos para cada espécie dos referidos primatas. Adicionalmente, para determinar a abundância das espécies, consideraram-se visualizações obtidas em trilhas acessórias. Obtevese uma densidade de 4,51 (IC = 2,40 - 8,48) grupos $/ \mathrm{km}^{2}$ para $C$. nigrifrons e de $7,45(\mathrm{IC}=3,82-14,54)$ grupos $/ \mathrm{km}^{2}$ para Callithrix sp. A abundância de C. nigrifrons correspondeu a 1,43 indivíduo/10 km percorridos e a de Callithrix sp., 1,17 indivíduo/10 km percorridos. O tamanho populacional estimado para C. nigrifrons foi de 28 indivíduos e para Callithrix sp., de 86. Por fim, esses resultados corroboram a necessidade de novas pesquisas, objetivando detectar flutuações populacionais ao longo do tempo, com o intuito de preservar e manejar essas espécies.
\end{abstract}

Palavras-chave: Primatas, densidade e tamanho populacional.

\section{DENSITY, POPULATION SIZE, AND ABUNDANCE OF PRIMATE POPULATIONS IN AN ATLANTIC FOREST FRAGMENT IN MINAS GERAIS, BRAZIL}

\begin{abstract}
The diversity of neo-tropical primates is represented by 128 species, with 26 threatened species, 24 of which are endemic of Brazil. Of these threatened primates, 15 species occur in the Atlantic Forest, indicating an alarming state of conservation due to anthropogenic factors. Mata do Paraíso, the largest fragment of this biome in Viçosa, Minas Gerais, comprises an area of 384.5 ha, whose primate population is represented by Callicebus nigrifrons (Spix, 1823), known by titi monkey, and Callithrix sp., denominated marmosets. The present study aimed at estimating the density and population size of these species, as well as at determining their abundance in the area. Five linear $1 \mathrm{~km}$ transects, placed in a parallel fashion in a representative portion of the forest, were covered by following the premises of the methodology Distance
\end{abstract}

\footnotetext{
${ }^{1}$ Recebido em 03.05.2007 e aceito para publicação em 22.08.2008.

${ }^{2}$ Graduanda em Ciências Biológicas pela Universidade Federal de Viçosa (UFV). E-mail: <evaniammsantana@yahoo.com.br>.

${ }^{3}$ Programa de Pós-Graduação em Ciência Florestal da UFV. E-mail: <marerp@ yahoo.com>.

${ }^{4}$ Departamento de Biologia Animal da UFV. E-mail: <gislessa@ yahoo.com.br>.

${ }_{5}^{5}$ Instituto de Ciências Biológicas da Universidade Federal de Goiás (UFG). E-mail: <frmelo@ carangola.br>.
} 
for linear transects. The data were collected from August 2004 to February 2006, with 70 samplings, totaling $82.7 \mathrm{~km}$ being sampled, with 24 primate detections. A density of 4.51 groups $/ \mathrm{km}^{2}$ for Callicebus nigrifrons and $7.45 \mathrm{groups} / \mathrm{km}^{2}$ for Callithrix sp was obtained. Callicebus nigrifrons' abundance corresponded to 1.43 individuals $/ 10 \mathrm{~km}$ sampled, and the abundance of Callithrix sp. corresponded to 1.17 individuals $/ 10 \mathrm{~km}$ sampled. The population size of Callicebus nigrifrons was estimated in 28 individuals and for Callithrix sp., in 86 individuals. This result corroborates the need for further research to detect fluctuations along time, aiming to preserve and manage these species.

Keywords: Primates, density and population size .

\section{INTRODUÇÃO}

Aproximadamente, $36 \%$ de toda a Ordem Primates, excluindo os Hominoidea, representada por 128 espécies distribuídas em quatro famílias, corresponde à diversidade de primatas neotropicais (WILSON e REEDER, 2005). Na Lista Oficial da Fauna Brasileira Ameaçada de Extinção (BRASIL, 2003) constam 26 espécies de primatas em categorias de ameaça, sendo 24 endêmicas do Brasil. Desses primatas ameaçados, 15 espécies ocorrem na Floresta Atlântica, onde sete estão criticamente em perigo, quatro em perigo e outras quatro vulneráveis (RYLANDS e CHIARELLO, 2003). O estado de conservação alarmante desses primatas reflete a severidade de fatores antrópicos atuando na Floresta Atlântica (JONES, 2001). De acordo com Estrada e Coates-Estrada (1996), a densidade de primatas tem diminuído significativamente devido à fragmentação florestal, resultando até mesmo em extinções locais. Cullen Júnior. et al. (2000) e Chiarello (2003) reforçaram que esse fenômeno de extinção é potencialmente aumentado pelos efeitos da caça em fragmentos florestais isolados, embora não seja tão evidente para primatas como o é para outros mamíferos terrestres de médio e grande porte, em particular veados (Mazama spp.) e antas (Tapirus terrestris (Linnaeus, 1758)), nos trechos de Floresta Atlântica estudados por esses autores.

Entre as três espécies de primatas enquadradas como quase ameaçadas de extinção na Floresta Atlântica (RYLANDS e CHIARELLO, 2003), de acordo com as categorias instituídas por Iucn (2001), encontra-se Callicebus nigrifrons (Spix, 1823), popularmente conhecida como sauá ou guigó. Este é um primata endêmico da Floresta Atlântica brasileira, podendo ocorrer também em matas ciliares do Cerrado (HERSHKOVITZ, 1988, 1990), estendendo-se pelos estados do Rio de Janeiro e de São Paulo e Sul de Minas Gerais (VAN ROOSMALEN et al., 2002). C. nigrifrons

R. Árvore, Viçosa-MG, v.32, n.6, p.1109-1117, 2008 é a maior das 28 espécies do gênero, atingindo até $2 \mathrm{~kg}$ quando adulto. Geralmente, vive em unidades familiares compostas por um casal monogâmico, um filhote e, às vezes, um jovem do ano anterior (KINZEY, 1981).

Originalmente, apenas 13 espécies de Callicebus eram consideradas válidas (HERSHKOVITZ, 1990). Kobayashi e Langguth (1999), ao descreverem uma nova espécie do gênero Callicebus, sugeriram uma nova classificação, adotada por Rylands et al. (2000) e Van Roosmalen et al. (2002), na qual essas 13 espécies foram validadas em 28 , divididas em cinco grandes grupos, sendo quatro amazônicos (donacophilus, torquatus, moloch, cupreus) e apenas um presente no leste do Brasil (personatus). Rodrigues et al. (2004) corroboram a validação das espécies do grupo personatus, ao diferenciar o número diplóide de duas das cinco espécies, C. nigrifrons e C. personatus (É. Geoffroy, 1812).

Quanto aos calitriquídeos, estes vivem em grupos de 4 a 15 indivíduos, alcançando 10 anos de vida na naturezae 16 anos em cativeiro(STEVENSON RYLANDS, 1988). Callithrix penicillata (E. Geoffroy, 1829) está distribuído pela costa brasileira, da Bahia a São Paulo e interior de Goiás, e C. geoffroyi (Humboldt, 1812) distribui-se no centro-leste do Brasil (VIVO, 1991; MENDES, 1997).

Poucas pesquisas abordando parâmetros populacionais de primatas, como densidade e abundância relativa, foram realizadas na Zona da Mata mineira (PEREIRA et al., 1995; OLIVEIRA et al., 2003), mas na Mata do Paraíso, maior fragmento florestal presente no Município de Viçosa, apenas um estudo sobre densidade populacional de $C$. nigrifrons foi conduzido (MELO, 1995). A espécie estudada por Melo (1995) foi de fato C. nigrifrons e não C. personatus, pois, por ocasião do estudo de Melo (1995), o grupo 
personatus era formado por apenas uma espécie ( $C$. personatus), dividida em quatro subespécies, incluindo Callicebus personatus nigrifrons (HERSHKOVITZ, 1990). Para Callithrix sp., o único trabalho precedente nessa região aborda aspectos morfológicos, caracterizando a população que habita a Mata do Paraíso como possíveis híbridos, resultantes do cruzamento entre $C$. geoffroyi e C. penicillata (MORAIS JÚNIOR, 1998).

Nos últimos anos, a identificação de mamíferos em campo, principalmente primatas, tem-se baseado na metodologia de transectos lineares (WHITESIDES et al., 1988; CHIARELLO e MELO, 2001; MARTINS, 2005), pois muitas espécies dessa ordem são conspícuas e de hábitos diurnos, facilmente detectáveis em trabalhos de campo (NRCCNP, 1981).

A metodologia de transectos lineares assume quatro premissas, em ordem decrescente de importância: (1) todos os animais posicionados diretamente na linha do transecto devem ser detectados; (2) todos os animais são detectados na sua posição inicial, antes de qualquer movimento em resposta ao observador; (3) as distâncias perpendiculares são medidas corretamente; e (4) as detecções são eventos independentes (BUCKLAND et al., 1993; THOMAS et al., 2002; CULLEN JÚNIOR e RUDRAN, 2003).

Transectos lineares distribuídos ao acaso podem ser preferíveis em áreas de floresta contínua de grandes extensões. No entanto, é mais apropriado conduzir uma forma de amostragem sistemática nos pequenos fragmentos ( $<500$ ha), como através de transectos paralelos, os quais podem se estender pela maior parte da área de estudo (PERES, 1999).

A região de estudo manteve-se praticamente inalterada até o início do século XIX, quando o café passou a se expandir pelo território mineiro. Hoje, a maior parte da cobertura vegetal dos fragmentos do Município de Viçosa é representada por Floresta Estacional Semidecidual fragmentada, devido ao histórico de desenvolvimento agrícola na região, principalmente da ocupação de áreas por pastos e plantações de café, realizada predominantemente por pequenos proprietários rurais familiares (PANIAGO, 1983).

Existem nove propriedades rurais no entorno imediato da Mata do Paraíso, ocupadas por moradores antigos, cujos proprietários demonstraram bom conhecimento sobre questões ambientais, não representando impacto direto à área (SANTANA, 2006). Porém, diversos relatos obtidos e observações pessoais demonstram forte impacto provocado pela caça, possivelmente de moradores distantes da área. Historicamente, a caça pode ter representado a extinção de primatas como o macaco-prego (Cebus nigritus(Goldfuss, 1809)) e o barbado (Alouatta clamitansCabrera, 1904), atualmente ausentes na área de estudo, mas ainda encontrados em outros fragmentos florestais dentro do Município de Viçosa e região (MELO, 1995).

Nesses termos, os objetivos deste estudo foram estimar a densidade e o tamanho populacional e avaliar a abundância relativa das duas espécies de primatas, C. nigrifrons e Callithrix sp., que ocorrem na Mata do Paraíso, em Viçosa, MG. Pretende-se, com os dados deste estudo, subsidiar futuros planos de manejo e conservação dessas populações, além de incentivar e mobilizar pessoas na execução de novas pesquisas.

\section{MATERIAL E MÉTODOS}

\section{1. Área de estudo}

O estudo foi realizado no fragmento florestal Mata do Paraíso (ponto central com as seguintes coordenadas geográficas: $20^{\circ} 48^{\prime} 06$ 'S e 4251'23'W), localizado em Viçosa, Zona da Mata mineira. Trata-se do maior fragmento florestal presente no município, com uma superfície de 384,5 ha (RIBON, 1998), dos quais 194 ha pertencem à Universidade Federal de Viçosa (UFV) e são utilizados para fins educativos e de pesquisa (ALMEIDA JÚNIOR, 1999; SILVA et al., 2004).

A altitude média da área de estudo é de $670 \mathrm{~m}$ e o relevo, montanhoso, com predominância de Latossolo Vermelho-Amarelo, no topo e nas encostas das elevações; e Podzólico Vermelho-Amarelo Câmbico, nos terraços (REZENDE, 1971; CORRÊA, 1984). A precipitação média anual na região é de $1.221 \mathrm{~mm}$, concentrada entre os meses de outubro e março, sendo a temperatura média anual de $19^{\circ} \mathrm{C}$ (VIANELLOe ALVES, 1991). O clima da região, segundo Köppen, é do tipo Cwb, tropical de altitude, com verões quentes e chuvosos e invernos frios e secos (ANTUNES, 1986).

\subsection{Coleta dos dados}

No período entre agosto de 2004 e fevereiro de 2006 , foram percorridos cinco transectos lineares de $1 \mathrm{~km}$ cada, dispostos de modo paralelo, com afastamento de $100 \mathrm{~m}$ entre eles, e trechos perpendiculares aos

R. Árvore, Viçosa-MG, v.32, n.6, p.1109-1117, 2008 
transectos. Os levantamentos corresponderam a caminhadas de 1,0 a $1,5 \mathrm{~km}$, realizadas em sua maioria por apenas um observador, numa velocidade média de $0,67 \mathrm{~km} / \mathrm{h}$. A coleta de dados foi conduzida em média três vezes por mês, sendo interrompida nos dias chuvosos e nos meses de julho e janeiro.

Foram realizados 70 levantamentos, 42 no período da manhã (das $7 \mathrm{~h}$ às $12 \mathrm{~h}$ ) e 28 à tarde (das $14 \mathrm{~h}$ às $18 \mathrm{~h}$ ), coincidindo com os horários de pico de atividade dos primatas (PERES, 1999), totalizando 82,7 km percorridos ao longo de $130 \mathrm{~h}$ de trabalhos em campo.

Em cada animal (no caso de indivíduos solitários) ou no primeiro indivíduo de cada grupo visualizado, foram anotadas as seguintes informações: distância perpendicular entre o animal detectado e o transecto ou o ângulo e distância do avistamento, transecto percorrido, tempo gasto no percurso, local e horário de cada avistamento, espécie visualizada e número de indivíduos por grupo. Nas detecções cujos ângulos $(\theta)$ e distâncias de avistamento (r) foram obtidos, a distância perpendicular ( $\mathrm{x}$ ) entre o animal detectado e o transecto foi calculada de acordo com a fórmula $\mathrm{x}=\mathrm{r}$. $\operatorname{sen} \theta$. As distâncias foram determinadas por meio de uma trena de $30 \mathrm{~m}$ e os ângulos, com uma bússola. Além disso, foi estimada a altura das árvores onde os primatas estavam quando avistados.

Adicionalmente, dados sobre a abundância dos primatas também foram gerados, a partir dos registros obtidos nos transectos lineares e em trilhas acessórias, durante percursos ao longo da área de estudo.

\subsection{Análise dos dados}

Para estimar a densidade e tamanho populacional dos primatas, a análise estatística foi conduzida utilizandose o software Distance 5.0, disponibilizado gratuitamente para download no site: http://www.ruwpa.st-and.ac.uk/ distance/ (THOMAS et al., 2005). O fundamento desse método é a busca de um modelo, ou uma Função de Detecção, que melhor espelhe o comportamento das distâncias perpendiculares observadas. Depois, utiliza-se essa função para estimar a proporção de indivíduos que não foram detectados durante os levantamentos, e, a partir daí, podese obter uma estimativa de densidade da população de interesse (BUCKLAND etal., 1993; THOMAS et al., 2002; CULLEN JÚNIOR e RUDRAN, 2003).

Simulações foram efetuadas com todos os modelos e ajustes disponíveis no software Distance 5.0, com dados truncados (descartando dados a partir de determinadas distâncias) e não truncados, e a escolha do modelo que se ajustou melhor aos dados ocorreu, levando-se em consideração a combinação de dois fatores: mínimo AIC (Akaike's Information Criterion) (THOMAS et al., 2002; CULLEN JÚNIOR e RUDRAN, 2003; JATHANNA et al., 2003) e menor coeficiente de variação (CV). O AIC é um índice para a seleção de modelo, sendo computado para cada modelo sob análise, e aquele que apresentar o menor valor para AIC é selecionado, atentando para identificar um modelo que seja adequado aos dados e que não tenha muitos parâmetros (BUCKLAND et al., 1993).

Observando os histogramas de distribuição das distâncias perpendiculares obtidas neste estudo e os resultados das simulações das análises de $C$. nigrifrons e Callithrix sp., optou-se por considerar todos os dados de distâncias perpendiculares, ou seja, não foi feita truncagem deles, à medida que acarretaria aumento no intervalo de confiança e no coeficiente de variação. O modelo e ajuste mais adequados aos dados de ambas as espécies foi half-normal + simple polynomial.

As densidades populacionais estão apresentadas em número de grupos por $\mathrm{km}^{2}$, acompanhadas de seus intervalos de confiança (IC) no nível de significância de 0,05 (95\% de probabilidade). Uma vez calculada a densidade populacional de grupos, esse valor foi multiplicado pelo tamanho médio dos grupos obtido neste estudo (número de indivíduos avistados dividido pelo número de avistamentos obtidos), para gerar o tamanho populacional da espécie na área de estudo.

A partir dos dados obtidos nos transectos e em trilhas acessórias, derivou-se o índice de abundância relativa de cada espécie de primata detectada, seguindo a metodologia utilizada por Chiarello e Melo (2001), Oliveira et al. (2003) e Bernardo e Galetti (2004). O índice de abundância indica o número de grupos de cada espécie encontrados a cada $10 \mathrm{~km}$ percorridos.

\section{RESULTADOS E DISCUSSÃO}

\subsection{Densidade e tamanho populacional}

Nos transectos lineares foram obtidas 24 visualizações de primatas, sendo 12 para cada espécie (C. nigrifrons e Callithrix sp.). Muito embora o número mínimo de detecções independentes recomendável seja 40, tamanhos amostrais menores também podem gerar 
estimativas robustas, dependendo da distribuição dos dados (BUCKLAND et al., 1993; PERES, 1999; CULLEN JÚNIOR e RUDRAN, 2003). Nesse sentido, deve ser considerado que nem sempre é obtido o número ideal de detecções dos animais, especialmente de espécies raras, o que não impede que sejam efetuadas estimativas confiáveis da densidade populacional, desde que os dados possibilitem o ajuste da Função de Detecção, com coeficiente de variação dentro de limites aceitáveis, fato que ocorreu neste estudo.

A partir dos dados de campo, foi estimada a densidade e o tamanho populacional das duas espécies de primatas que vivem na Mata do Paraíso, conforme apresentado nos tópicos subseqüentes.

\subsubsection{Callicebus nigrifrons}

O tamanho médio dos grupos encontrado em $C$. nigrifrons foi de 1,63 indivíduo/grupo. Resultado semelhante foi registrado por Bernardo e Galetti (2004) em área de Floresta Atlântica no Estado de São Paulo, com superfície de 230 ha, onde foi estimado o tamanho médio de grupos de 1,71 indivíduo/grupo. No entanto, num estudo realizado anteriormente na Mata do Paraíso o tamanho médio de grupos encontrado foi de 3,68 indivíduos/grupo (MELO, 1995), aproximando-se do valor de 3,09 indivíduos/grupo obtido por Oliveira et al. (2003) em outro fragmento de 75 ha de Floresta Atlântica, também em Viçosa, MG. Essas divergências podem estar relacionadas às diferenças metodológicas no que se refere à coleta de dados para obtenção do tamanho médio dos grupos e, ou, ao período de realização de cada estudo, à medida que durante a fase de criação dos filhotes os grupos familiares são maiores. Além disso, podem advir de variações ambientais inerentes a cada fragmento, como tempo de perturbação e densidade de lianas, entre outras não mensuradas aqui.

A densidade populacional estimada para $C$. nigrifrons foi de 4,51 (IC = 2,40 - 8,48) grupos $/ \mathrm{km}^{2}$ (Quadro $1)$, valor semelhante ao encontrado por Oliveira et al.
(2003), cuja estimativa gerou uma densidade de 4,80 grupos $/ \mathrm{km}^{2}$. Dessa forma, considerando o tamanho médio de grupos de 1,63 indivíduo/grupo, encontrado neste estudo, obtém-se uma densidade populacional de 7,35 indivíduos $/ \mathrm{km}^{2}$. Resultado similar (6,4 indivíduos/ $\mathrm{km}^{2}$ ) foi obtido para C. personatus numa área de 210 ha de Floresta Atlântica no Estado do Espírito Santo (CHIARELLO e MELO, 2001).

Como o fragmento amostrado estendia-se por 3,84 $\mathrm{km}^{2}$, o tamanho da população de $C$. nigrifrons foi estimado em cerca de 28 indivíduos. Esse valor é relativamente menor que o obtido por Melo (1995), cujas estimativas apontaram a existência de 35 a 52 indivíduos (13 grupos) dessa espécie, em 194 ha da Mata do Paraíso. Vale destacar que a análise feita por Melo (1995) levou em conta 194 ha de área para efeito de cálculo de densidade populacional, mas a área efetiva da Mata do Paraíso, mesmo naquela ocasião, era de aproximadamente 384 ha. Isso implica uma observação de que pudessem existir ainda mais grupos de $C$. nigrifrons na Mata do Paraíso, aumentando ainda mais a diferença entre a densidade aqui diagnosticada. Como a densidade observada por Melo (1995) foi de 27 indivíduos $/ \mathrm{km}^{2}$, refazendo os cálculos poderiam existir 104 indivíduos em 28 grupos, ao considerar a área de $3,84 \mathrm{~km}^{2}$ e o tamanho médio de grupo em 3,68 indivíduos (MELO, 1995). Esses valores seriam bem superiores aos encontrados nesta pesquisa, mas reforçam as diferenças metodológicas, podendo refletir mais um artefato de técnica do que propriamente uma diferença real.

\subsubsection{Callithrix sp.}

O tamanho médio dos grupos encontrado em Callithrix sp. foi de 3,00 indivíduos/grupo. Resultados parecidos foram obtidos em C. aurita (E. Geoffroy in Humboldt, 1812), em áreas de Floresta Atlântica no Estado de São Paulo, por Bernardo e Galetti (2004), que encontraram 3,14 indivíduos/grupo, e por Martins (2005), 3,7 indivíduos/grupo.

Quadro 1 - Estimativa da densidade populacional de Callicebus nigrifrons e de Callithrix sp. na Mata do Paraíso, Viçosa, MG Table 1 -Density estimate of Callicebus nigrifrons and Callithrix sp. in the Mata do Paraíso, Viçosa, MG

\begin{tabular}{|c|c|c|c|c|c|}
\hline Espécie & $\mathrm{N}$ & Densidade (grupos/km²) & Erro padrão & $\mathrm{CV}(\%)$ & $\mathrm{IC}$ \\
\hline Callicebus nigrifrons & 12 & 4,51 & 1,46 & 32,27 & $2,40-8,48$ \\
\hline Callithrix sp. & 12 & 7,45 & 2,55 & 34,29 & $3,82-14,54$ \\
\hline
\end{tabular}

$\mathrm{N}=$ número de visualizações; $\mathrm{CV}=$ coeficiente de variação e $\mathrm{IC}=$ intervalo de confiança, com probabilidade de $95 \%$.

$\mathrm{N}=$ number of detections; $\mathrm{CV}=$ coefficient of variation and $\mathrm{CL}=$ confidence limits with probability of $95 \%$. 
A densidade populacional estimada para Callithrix sp. na área de estudo foi de 7,45 (IC = 3,82 - 14,54) grupos $/ \mathrm{km}^{2}$ (Quadro 1). Considerando o tamanho médio de 3,00 indivíduos/grupo, obteve-se uma densidade populacional de 22,35 indivíduos $/ \mathrm{km}^{2}$. Valores inferiores foram detectados em outros estudos, como de Bernardo e Galetti (2004), que registraram uma densidade de 3,5 indivíduos $/ \mathrm{km}^{2}$ para C. aurita, numa área de 230 ha de Floresta Atlântica, no Estado de São Paulo. De forma semelhante, Chiarello e Melo (2001) encontraram uma densidade de 3,8 indivíduos $/ \mathrm{km}^{2}$ para $C$. geoffroyi, numa área de 210 ha de Floresta Atlântica, no Estado do Espírito Santo.

Considerando que o fragmento amostrado estendese por $3,84 \mathrm{~km}^{2}$, foi estimado o tamanho populacional de Callithrix sp. em aproximadamente 86 indivíduos. Dessa forma, tanto a densidade quanto o tamanho populacional encontrados neste estudo para Callithrix sp. podem ser considerados relativamente elevados, fato que deve ter ocorrido em função de a população em questão ser híbrida e, por isto, é provável que apresente maior adaptabilidade e resistência (COIMBRAFILHO et al., 1984, 1993). Além disso, a área amostrada possui em seu histórico a soltura indiscriminada de indivíduos desse gênero, colaborando para o aumento de sua população (SANTANA et al., 2006). Apesar de não haver números concretos, relatos contundentes dessa soltura aleatória foram obtidos com freqüência ao longo dos anos de estudo (F. R. Melo, observação pessoal).

Estudos tanto de estimativas de densidade quanto de tamanho populacional indicaram a presença de menos de 50 espécimes de diferentes primatas em fragmentos florestais pequenos (com aproximadamente 200 ha) (CHIARELLO e MELO, 2001). Segundo esses autores, tal fato pode estar relacionado à redução de espaço, menor disponibilidade de recursos alimentares e alta pressão de predadores de médio porte, porém áreas maiores tendem a apresentar densidade populacional mais elevada. Nesse sentido, Mendes Pontes (1999) observou que a variável mais importante na determinação da abundância de primatas em cada tipo de ambiente é a disponibilidade de alimento, a qual, associada com outros fatores ambientais, determina quais espécies que têm acesso a uma fonte alimentar mais abundante e podem, conseqüentemente, alcançar densidades populacionais maiores.

Deve se considerar que os fragmentos florestais, ainda que se apresentem parcialmente isolados, são

R. Árvore, Viçosa-MG, v.32, n.6, p.1109-1117, 2008 importantes mantenedores da biodiversidade (PEREIRA et al., 2001). Nesse sentido, a conservação da Mata do Paraíso é de suma importância, pois, além de ser o maior fragmento florestal do Município de Viçosa, MG, funciona como área de refúgio num ambiente com muita influência antrópica, apesar de existir evidência de caça. Além disso, espécies de primatas de menor porte (Callithrix spp. e Callicebus spp.) são menos visadas para caça esportiva, apesar de serem eventualmente predadas por cães e procuradas como "pets" (CHIARELLO, 2003).

\subsection{Abundância}

A partir dos dados obtidos nos transectos e em trilhas acessórias, totalizando $112 \mathrm{~km}$ percorridos, derivou-se um índice de abundância relativa para cada espécie, e $C$. nigrifrons apresentou maior abundância de grupos (1,43 grupo/10 km percorridos) que Callithrix sp. (1,17 grupo/10 km percorridos). Esse resultado diverge daquele encontrado, com relação à densidade populacional, neste estudo, fato que pode ser explicado pelo tamanho corporal de cada uma das espécies em questão, uma vez que indivíduos de maior porte são freqüentemente mais visualizados.

Valores diferentes são registrados em outras áreas de Floresta Atlântica com grupos de pequenos primatas, como visto em Bernardo e Galetti (2004) numa área de 230 ha, no Estado de São Paulo, cuja abundância foi de 0,56 grupo $/ 10 \mathrm{~km}$ percorridos tanto com relação a C. nigrifrons quanto a C. aurita. Já Chiarello e Melo (2001), estudando C. personatus, registraram 1,02 grupo/ $10 \mathrm{~km}$ percorridos, num fragmento de $210 \mathrm{ha}$, no Estado do Espírito Santo.

\subsection{Altura das árvores}

A altura média estimada de árvores em que se visualizou $C$. nigrifrons foi de $15,67 \mathrm{~m}$ e a daquelas que continham Callithrix sp., 4,67 m. Da mesma forma, Oliveira et al. (2003) registraram altura média de $18 \mathrm{~m}$ nas detecções de C. nigrifrons, inferindo que essa espécie apresenta preferência pelo estrato superior da mata.

\section{CONCLUSÕES}

A densidade populacional estimada de $C$. nigrifrons foi de 4,51 (IC = 2,40-8,48) grupos $/ \mathrm{km}^{2}$, resultando numa população de aproximadamente de 28 indivíduos na Mata do Paraíso. 
A densidade populacional estimada de Callithrix sp. foi de 7,45 (IC = 3,82 - 14,54) grupos $/ \mathrm{km}^{2}$, resultando numa população de cerca de 86 indivíduos.

A abundância relativa encontrada em C. nigrifrons foi de 1,43 grupo/10 km percorridos e em Callithrix sp., 1,17 grupo/10 km percorridos.

A altura média estimada das árvores onde se visualizaram $C$. nigrifrons foi de $15,67 \mathrm{~m}$ e a das que continham Callithrix sp., 4,67 m, indicando que a primeira espécie apresenta preferência pelo estrato superior da mata e, a segunda, pelo estrato intermediário.

Com base nos resultados deste estudo, recomendamse novas pesquisas sobre densidade e estrutura populacional de primatas na Mata do Paraíso, entre outros, visando detectar flutuações populacionais ao longo do tempo e entre as estações do ano. Além disso, é desejável que trabalhos comportamentais sejam realizados, indicando estimativas mais precisas sobre tamanhos médios dos grupos de primatas e sua distribuição ao longo da área.

\section{AGRADECIMENTOS}

Especialmente ao Professor Renato Neves Feio, pelo incentivo desde o início do projeto; a Andressa Sales Coelho e Regiane Romanini de Oliveira, pelas valiosas sugestões; e aos Srs. Zé Brás, Lair, Vanderli e Fernando pela importante ajuda na abertura dos transectos.

\section{REFERÊNCIAS}

\author{
ALMEIDA JÚNIOR, J. S. Florística e \\ fitossociologia de fragmentos da \\ floresta estacional semidecidual. 1999. \\ 148f. Dissertação (Mestrado em Ciência \\ Florestal) - Universidade Federal de Viçosa, \\ Viçosa, MG, 1999.
}

ANTUNES, F. Z. Caracterização climática do estado de Minas Gerais. Informe Agropecuário, v.12, n.138, p.9-13, 1986.

BERNARDO, C. S. S.; GALETTI, M. Densidade e tamanho populacional de primatas em um fragmento florestal no sudeste do Brasil.

Revista Brasileira de Zoologia, v.21, n.4, p.827-832, 2004.

BRASIL. Ministério do Meio Ambiente. Lista das espécies da fauna brasileira ameaçadas de extinção. Instrução Normativa, n.3, de 27 de maio de 2003. Brasília: 2003.
BUCKLAND, S. T. et al. Distance sampling; estimating abundance of biological populations. London: Chapman \& Hall, 1993. 446p.

CHIARELLO, A. G. Primates of the Brazilian Atlantic Forest: the influence of forest fragmentation on survival. In: MARSH, L. K. (Ed.) Primates in fragments. New York: Kluwer Academic/Plenum Publishers, 2003. p.99-122.

CHIARELlO, A. G.; MELO, F. R. Primate population densities and sizes in Atlantic forest remnants of northern Espírito Santo, Brazil.

International Journal of Primatology, v.22, n.3, p.379-396, 2001.

COIMBRA-FILHO, A. F.; SILVA, R. R.; PISSINATTI, A. Heterose em fêmea híbrida de Callithrix. (Callitrichidae-Primates). In: MELLO, M. T. (Ed.) A primatologia no Brasil. Salvador: Sociedade Brasileira de Primatologia, 1984. p.213-216.

COIMBRA-FILHO, A. F.; PISSINATTI, A.; RYLANDS, A. B. Experimental multiple hybridism and natural hybrids among Callithrix species from eastern Brazil. In: RYLANDS, A. B. (Ed.) Marmosets and Tamarins: systematics, behaviour and ecology. New York: Oxford University Press, 1993. p.95-122.

CORRÊA, G. F. Modelo de evolução mineralógica da fração argila de solos do planalto de Viçosa. 1984. $187 \mathrm{f}$. Dissertação (Mestrado em Ciência Florestal) Universidade Federal de Viçosa, Viçosa, MG, 1984.

CULLEN JÚNIOR, L.; RUDRAN, R. Transectos lineares na estimativa de densidade de mamíferos e aves de médio e grande porte. In: CULLEN JÚNIOR, L.; RUDRAN, R.; VALLADARESPÁDUA, C. (Orgs.) Métodos de estudos em biologia da conservação e manejo da vida silvestre. Curitiba: Universidade Federal do Paraná, 2003. p.169-179.

CULLEN JÚNIOR.,L.; BODMER, R. E.; VALLADARESPÁDUA, C. Effects of hunting in habitat fragments of the Atlantic Forests, Brazil. Biological Conservation, v.95, n.1, p.49-56, 2000.

ESTRADA, A.; COATES-ESTRADA, R. Tropical rain forest fragmentation and wild populations of primates at Los Tuxtlas, México. International Journal of Primatology, v.17, n.5, p.759-783, 1996.

R. Árvore, Viçosa-MG, v.32, n.6, p.1109-1117, 2008 
HERSHKOVITZ, P. Origin, speciation, and distribution of South American titi monkeys, genus Callicebus (family Cebidae, Platyrrhini). Proceedings of the Academy of Natural Sciences of Philadelphia, v.140, n.1, p.240-272, 1988.

HERSHKOVITZ, P. Titis, New World monkeys of the genus Callicebus (Cebidae, Platyrrhini): A preliminary taxonomic review. Fieldiana, Zoology, New Series, v.55, p.1-109, 1990.

The word conservation union - IUCN. IUCN Red list categories and criteria: version 3.1. IUCN Species survival commission. IUCN: Gland, Switzerland and Cambridge, U.K., 2001. 30p.

JATHANNA, D.; KARANTH, K. U.; JOHNSINGH, A. J. T. Estimation of large herbivore densities in the tropical forests of southern India using distance sampling. Journal of Zoology, v.261, n.3, p.285-290, 2003.

JONES, C. B. Introduction: Sampling Neotropical Primates: implications for conservation and socioecology. Primate Report, v.61, p.67, 2001.

KINZEY, W. G. The Titi monkeys, genus Callicebus. In: COIMBRA-FILHO, A. F.; MITTERMEIER, R. A. (Eds.) Ecology and behavior of neotropical primates. Rio de Janeiro: Academia Brasileira de Ciências, 1981.p.241-276. v.1.

KOBAYASHI, S.; LANGGUTH, A. A new species of titi monkey, Callicebus Thomas, from north-eastern Brazil (PRIMATES, CEBIDAE). Revista Brasileira de Zoologia, v. 16, n.2, p.531-551, 1999.

MARTINS, M. M. Density of primates in four semi-deciduous forest fragments of São Paulo, Brazil. Biodiversity and Conservation, v.14, n.10, p.2321-2329, 2005.

MELO, F. R. Densidade, tamanho e composição de grupos de Callicebus personatus (CEBIDAE: PRIMATES) em fragmentos florestais do município de Viçosa, MG. 1995. 29f. Monografia (Graduação em Ciências Biológicas) - Universidade Federal de Viçosa, Viçosa, MG, 1995.

R. Árvore, Viçosa-MG, v.32, n.6, p.1109-1117, 2008
MENDES PONTES, A. R. Environmental determinants of primate abundance in Maracá Island, Roraima, Brazilian Amazônia. Journal of Zoology, v.247, p.189-199, 1999.

MENDES, S. L. Padrões biogeográficos e vocais em Callithrix do grupo jacchus. 1997. 80f. Tese (Doutorado em Ecologia) - Universidade Estadual de Campinas, Campinas, 1997.

MORAIS JÚNIOR., M. Aspectos ecológicos e morfológicos de um grupo social de possíveis híbridos de Callithrix (CALLITHRICHIDAE; PRIMATES) em Viçosa, MG. 1998. 25f. Monografia (Graduação em Ciências Biológicas) - Universidade Federal de Viçosa, Viçosa, MG, 1998.

\section{NATIONAL RESEARCH COUNCIL COMMITTEE} ON NONHUMAN PRIMATES - NRCCNP.

Techniques for the study of primate population ecology. New York: National Academy Press, 1981. p.233.

OLIVEIRA, R. R.; COELHO, A. S.; MELO, F. R. Estimativa de densidade e tamanho populacional de Sauá (Callicebus nigrifrons) em um fragmento de mata em regeneração, Viçosa, Minas Gerais, Brasil. Neotropical Primates, v.11, n.2, p.91-94, 2003.

PANIAGO, M. C. T. Evolução histórica e tendências de mudanças sócioculturais na comunidade de ViçosaMG. 1983. 407f. Dissertação (Mestrado em Educação) - Universidade Federal de Viçosa, Viçosa, MG, 1983.

PEREIRA, R. F. et al. Primates from the vicinity of Viçosa, Minas Gerais, Brazil. Neotropical Primates, v.3, n.4, p.171-173, 1995.

PEREIRA, R. A. et al. Caracterização da paisagem, com ênfase em fragmentos florestais, do município de Viçosa, Minas Gerais. Revista Árvore, v.25, n.3, p.327-333, 2001.

PERES, C. A. General guidelines for standardizing line-transect surveys of tropical forest primates. Neotropical Primates, v.7, n.1, p.11-16, 1999. 
PERES, C. A. Synergistic effects of subsistence hunting and habitat fragmentation on Amazonian forest vertebrates. Conservation Biology, v.15, n.6, p.1490-1505, 2001.

REZENDE, S. B. Estudo de cronosequência em Viçosa - Minas Gerais. 1971. 71f. Dissertação (Mestrado em Fitotecnia) Universidade Federal de Viçosa, Viçosa, MG, 1971.

RIBON, R. Fatores que influenciam a distribuição da avifauna em fragmentos de Mata Atlântica nas montanhas de Minas Gerais. 1998. $127 \mathrm{f}$. Dissertação (Mestrado em Ecologia, Conservação e Manejo de Vida Silvestre) - Universidade Federal de Minas Gerais, Belo Horizonte, 1998.

RODRIGUES, L. R. R. et al. A new karyotype of an endangered primate species (Callicebus personatus) from the Brazilian Atlantic forests. Hereditas, v. 140, n.1, p.87-91, 2004.

RYLANDS, A. B.; CHIARELLO, A. Official list of brazilian fauna threatened with extinction 2003. Neotropical Primates, v.11, n.1, p.43-49, 2003.

RYLANDS, A. B. et al. An assessment of the diversity of New World primates. Neotropical Primates, v.8, n.2, p.61-93, 2000.

SANTANA, B. E. M. M. Densidade e tamanho populacional de sauás Callicebus nigrifrons (Spix, 1823) (Primates: Pitheciidae) e de sagüis Callithrix sp. (Erxleben, 1777) (Primates: Callitrichinae) em um fragmento de Mata Atlântica de Minas Gerais. 2006. 25f. Monografia (Graduação em Ciências Biológicas) - Universidade Federal de Viçosa, Viçosa, MG, 2006.

SANTANA, B. E. M. M.et al. Ocorrência de Callithrix jacchus na Mata do Paraíso, em Viçosa, MG. In: SIMPÓSIO DE INICIAÇÃO CIENTÍfICA. Viçosa, MG: Universidade Federal de Viçosa, 2006. CD-ROM

SILVA, C. T. et al. Avaliação temporal da florística arbórea de uma floresta secundária no município de Viçosa, MG. Revista Árvore, v.28, n.3, p.429-441, 2004.
STEVENSON, M. F.; RYLANDS, A. B. The marmosets, Genus Callithrix. In: MITTERMEIER, R. A. et al. (Eds.) Ecology and behavior of Neotropical Primates. Washington: 1988. v.2.p.131-222.

THE WORLD CONSERVATION UNION - IUCN. IUCN Red list categories and criteria: version 3.1. Cambridge: IUCN Species Survival Commission, 2001. 30p.

THOMAS, L. et al. Distance sampling. In: ELSHAARAWI, A. H.; PIEGORSCH, W. W. (Eds.) Encyclopedia of Environmetrics. Chichester: John Wiley \& Sons, 2002. v.1. p.544-552.

THOMAS, L. et al. Distance 5.0. Release “x"1. Research Unit for Wildlife Population Assessment, University of St. Andrews, UK. 2005. Disponível em: <http://www.ruwpa.stand.ac.uk/distance/>. Acesso em: 05.de março de 2006 .

van ROOSMALEN, M. G. M.; van ROOSMALEN, T.; MITTERMEIER, R. A. A taxonomic review of the titi monkeys, genus Callicebus Thomas, 1903, with the description of two new species, Callicebus bernhardi and Callicebus stephennashi, from Brazilian Amazônia. Neotropical Primates, v.10, p.1-52, 2002. Supplement.

VIANELLO, R. L.; ALVES, A. R. Meteorologia básica e aplicações. Viçosa, MG:

Universidade Federal de Viçosa, 1991. 449p.

\section{VIVO, M. Taxonomia de Callithrix}

Erxleben, 1777 (Callithrichidae, Primates). Belo Horizonte: Líttera Maciel; Fundação Biodiversitas para a Conservação da Diversidade Biológica, 1991. 105p.

WHITESIDES, G. H. et al. Estimating primate densities from transects in a West African rainforest: a comparison of techniques. Journal of Animal Ecology, v.57, p.345-367, 1988.

WILSON, D. E.; REEDER, D. M. (Eds.). Mammal species of the world: a taxonomic and geographic reference. 3.ed. Baltimore: The Johns Hopkins University Press, 2005. v.1.743p.

R. Árvore, Viçosa-MG, v.32, n.6, p.1109-1117, 2008 